\title{
ASSESSING AND IMPROVING THE COVERAGE OF A STRATEGIC RESEARCH AGENDA: A DESIGN THEORY APPROACH
}

\author{
Rémondeau, Estelle (1,2); Cogez, Patrick (2); Le Masson, Pascal (1); Weil, Benoît (1) \\ 1: MINES ParisTech - PSL Research University, France; 2: AENEAS, France
}

\begin{abstract}
Strategic Research Agendas (SRA) bring to the research community a prospective and collective vision of a sector and are intended to provide directions for future research efforts. However, some promising innovative areas are not always foreseen in those documents, which raises the question of the relevance and adequacy of their coverage. While engineering design is often considered to translate SRA guidelines into product development, we believe it can also be of great help regarding the design of an SRA. In this paper, we will first address how to assess the scope of an SRA through a framework based on C-K theory, before exploring how to extend it, if need be. To answer those questions, we will examine a high-quality roadmap: the Electronic Components and Systems Strategic Research Agenda (ECS SRA). Our resulting method will provide us the means to assess SRA coverage and to ensure that interesting research areas are not forgotten unintentionally, in order to allow to a further enrichment of the document if needed.
\end{abstract}

Keywords: Strategic Research Agenda, Evaluation, Design engineering, Design theory, C-K theory

\section{Contact:}

Rémondeau, Estelle

MINES ParisTech - PSL Research University

Center for Management Science - CGS i3 UMR CNRS 9217

France

estelle.remondeau@mines-paristech.fr 


\section{INTRODUCTION}

Numerous Strategic Research Agendas (SRA's) and roadmaps have been widely introduced in different fields to support both Research \& Innovation and business objectives (Barker and Smith, 1995). Through their structured insight of collective research priorities, they provide decision-makers with a clear understanding of a sector while identifying new opportunities or issues. Nevertheless, in some scientific domains, research efforts were proven to be concentrated in a limited number of areas (Agogué and Cassotti, 2012) and did not tackle all the possible desirable innovation options. As a result some agendas could miss some promising disruptive innovations. In this paper, we propose a methodology, based on a design framework, to diagnose whether there are interesting research areas that are not covered by Strategic Research Agendas (SRA). In addition, we describe how to identify non-stated concepts that could benefit from further investigation.

\section{LITERATURE REVIEW AND RESEARCH QUESTIONS}

The term Strategic Research Agenda (SRA) is widely and commonly used, but its distinctive character regarding the "roadmap" appellation stays imprecise. Even more so as there is no standard meaning or definition of what is covered by the designation of science and technology roadmaps (Lee and Park, 2005). Although they all share an extended look at the future of a chosen field of inquiry, and mobilize collective knowledge and imagination of drivers of change in the involved field (Galvin, 1998), many different types of roadmaps exist and have spread to diverse environments since their initial implementation (Willyard and McClees, 1997). Existing literature has attempted to classify them into various categories and conveniently SRA's echo some of Phaal and al. (2004) classifications such as text format and long-range planning. Therefore our definition of SRA's rely on those classifications and we consider an SRA as a text-based roadmap aiming at a strategic long-range collective vision of sectoral and multiorganizational environment, often designed to align national or international research endeavours. In that respect, SRA's define what are the priorities to be addressed collectively in a given research area, in order to gain knowledge and be able to tackle current and future issues, while extending the horizon of a domain. Furthermore, SRA's are sometimes the cornerstone for the calls for projects for different national or international funding programmes.

Literature has mainly considered the functions and uses of roadmaps, but rarely examines their assessment, as mentioned in the work of Robinson and Propp (2008). Actually, that kind of document can be evaluated according to varied requirements as the competence of the roadmap team, the relevance of stakeholders (Kostoff and Schaller, 2001), national priorities and many other criteria. In this article we will only focus on SRA assessment in terms of coverage, without taking into account other conditions. As a matter of fact, SRA's are supposed to focus on strategic collective priorities, and might not be entirely exhaustive in their coverage. Nevertheless, SRA writers must decide intentionally to omit some areas. In order to ensure the correct decisions are made, the widest possible coverage must be initially considered, without concentrating too much on a dominant design that encompasses the most commonly envisaged solutions for the ecosystem. The SRA elaboration process must foster a variety of potential paths in order to spur innovation, inspire the community and counterbalance the tendency of organisations to have restricted research horizons (Rosenberg, 1976). The resulting SRA will go beyond the path-dependency (David, 1985) of its source community and will complement it with a collection of promising paths creation (Garud \& Karnoe, 2001) which aims to deviate from commonly considered solutions.

Along this line, some communities have considered design theories to assess rigorously the ability of actors to break those dominant designs, unveiling promising unidentified paths. They include Cogez and al. (2013) work using C-K theory (Hatchuel and Weil, 2009) to prove the good coverage of the International Technology Roadmap for Semiconductors (ITRS). Their methodology enabled them to depict and assess the different paths described in the roadmap. Moreover, in a design perspective, SRA's must clearly distinguish acquired knowledge from identified unknowns which are relevant to be investigated in the future. In that respect, our first research question will address how to diagnose whether there are interesting research areas that are unintentionally not covered by SRA, through a tool differentiating clearly what is presently known and what is still undiscovered. 
Besides, such a method would be also useful to pilot exploration and propose additional relevant concepts to investigate. Indeed, C-K theory was also leveraged to build some referential frameworks allowing to distinguish unexplored pathways, leading to a potential enrichment of the research environment. This tool enabled Agogué and al. (2012) to unveil paths-in-the-unknown, only attainable through new innovation capabilities of involved actors. In that respect, our second research question will be how to create a method, that will allow the possibility to identify and investigate undiscovered promising innovation paths to potentially supplement an SRA, if need be? To answer those questions, we propose to develop a methodology based on a $\mathrm{C}-\mathrm{K}$ framework leading to SRA coverage assessment and enrichment.

\section{METHOD}

We propose to analyse an SRA content, through a design framework inspired by $\mathrm{C}-\mathrm{K}$ theory, transcribing the document content into a $\mathrm{C}-\mathrm{K}$ referential diagram. Those $\mathrm{C}-\mathrm{K}$ representations have been used in many studies as in the work of Cogez and al. (2011), Chen and al. (2017), Le Masson and al. (2017) or Vourc'h and al. (2018). However, this method must be adapted to our specific material, namely an SRA. Unlike previous studies, we do not have any initial concept to start with, but rather a text-based document, listing different topics and issues. SRA's are composed of numerous statements, paragraphs, often organized under sub-titles and major challenges in a chapter structure but may not distinguish clearly the concept space and the knowledge space defined in C-K theory. Hence, eventual reformulations may be needed to create proper conceptual formulations according to $\mathrm{C}-\mathrm{K}$ theory definition. Those formulations are explicit concepts (ECi) as they refer to SRA explicit content, this is also the case for their related knowledge (EKi). Each title or bullet point is thus transcribed into an $\mathrm{ECi}$, and SRA paragraphs are transcribed into local concept-tree composed of numerous ECi, while in the meantime their related knowledge is classified in a related basis.

\begin{tabular}{|c|c|c|c|c|c|}
\hline \multicolumn{4}{|c|}{ Base data from SRA content } & \multirow{4}{*}{\multicolumn{2}{|c|}{ 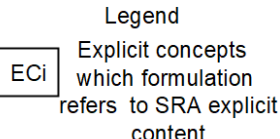 }} \\
\hline Standalone sentence : ti & oullet points $\mathrm{e}$ & \multirow{2}{*}{\multicolumn{2}{|c|}{ Paragraph }} & & \\
\hline k & Transcri & & & & \\
\hline Conceptual formulation & Knowledge & Conceptual formulation & Knowledge & & \\
\hline $\mathrm{ECi}$ & EKi & ECi & $\begin{array}{l}-E K i \\
-E K i \\
-E K i \\
-E K i\end{array}$ & $\frac{\sqrt{\mathrm{P}} \overline{\mathrm{Ci}}}{\mathrm{EKi}}$ & $\begin{array}{l}\text { Potential concepts } \\
\text { Explicit } \\
\text { knowledge }\end{array}$ \\
\hline
\end{tabular}

Figure 1. Method to transcribe SRA content into concepts and knowledge, while identifying the position of potential conceptual paths that are not covered by the document

In some situations, explicit concepts do not cover all the potential scope of a partition, and additional potential concepts (PCi) could be thus added in local trees. Those locations are clearly pinpointed during the transcription as such concepts prove that the document does not cover all the C-space. In order to have a broader analysis, we propose to collect all the ECi from standalone concepts or local trees and then, to arrange them into a general architecture, while the knowledge basis is structured into several pockets of knowledge, according to $\mathrm{C}-\mathrm{K}$ theory principles. However, ECi's resulting from SRA's are very unlikely to arrange into a perfect concept tree, as the SRA structure is not built according to this pattern. That is why, to organize such a tree we look for implicit concepts (ICi), present in the underlying content of the SRA in order to create a coherent architecture. Those common concepts help to structure the conceptual tree while putting in evidence common points that are shared by SRA items, but not stated explicitly. They enable the creation of structured innovation pathways corresponding to the SRA content and to identify further uncovered areas (PCi). Exchanges with SRA contributors help to ensure the coherence of the resulting diagram with the SRA content.

As a second step, we incorporate further knowledge from experts to make the referential architecture more robust and potentially find relevant paths to investigate. This knowledge can come from SRA contributors, experts of the involved field or experts from other communities. To that effect, three different methods are developed in this study:

1. Investigating potential concepts (PCi) identified through partition completion during the transcription phase

2. Adding knowledge coming from various communities to ECS SRA Explicit Concepts (ECi)

3. Generating new concepts to complete the ECS SRA scope 


\section{MATERIAL}

To apply our methodology, we looked for material written by numerous and highly qualified experts during a well-structured writing process. This document must deal with a very wide research scope and encompass diverse Technology Readiness Levels. We also sought a context where SRA authors were available to discuss with us the relevance of our approach. Thus, we chose to study the Electronic Components and Systems Strategic Research Agenda 2019 (ECS SRA) that spans the entire value chain of the electronics industry, covering Technology Readiness Level from 2 to 8, while setting out a single and common vision for Europe. Published by three European Industry associations AENEAS, ARTEMIS-IA and EPOSS and incorporating the work of over 200 experts across Europe, this document results from a regular writing process spread over a one-year period. Besides, most of authors were even more proficient as they were already familiar with roadmap writing process as those documents have been used in the electronic industry for many years. The first roadmap implementation was indeed conducted in Motorola (Willyard and McClees, 1997). Soon after the International Technology Roadmap for Semiconductor (ITRS) enterprise was launched, this time involving European actors. All those factors contribute a priori to create a very robust ECS SRA with the least possible bias. Besides, the ECS SRA is a cornerstone for the calls for projects of different European programmes such as ECSEL (Electronic Components and Systems for European Leadership) or EURIPIDES ${ }^{2}$ and PENTA, which are EUREKA clusters. Since this document is used as a reference for project creation, we will use the mapping of project proposals onto the Concept-trees that we will have developed as a control tool of our methodology. Besides, as the ECS SRA is structured in 5 key application chapters and 5 essential capabilities chapters, we proposed, in order to have a better sample variety, to focus our study both on an application chapter, about Transport and Smart Mobility, and on an essential capabilities chapter, about ECS Process Technology, Equipment, Materials and Manufacturing. Each ECS SRA chapter is structured around several Major Challenges which will help us to articulate our analysis. One researcher was fully employed in the AENEAS Association for a period of 6 months to lead the study. This position allowed the researcher to work in strong interactions with both ECS SRA contributors and programme managers to ensure the elaboration of relevant diagrams and adequate project proposals positioning. Furthermore, it allows the possibility to have access to knowledge from several experts from different SRA scientific domains to constantly improve diagrams and enrich them.

\section{ECS SRA ANALYSIS THROUGH C-K THEORY}

\subsection{Building a C-K roadmap diagnostic tool without any added knowledge}

We apply the first steps of our methodology to the chosen application chapter of the ECS SRA using C-K theory, as illustrated in Figure 2. First, we will start our analysis with Major Challenge 3 and all of its content. Standalone sentences are eventually reformulated into concepts, without adding any further knowledge to keep as closely as possible to the ECS SRA structure, most of the time adding

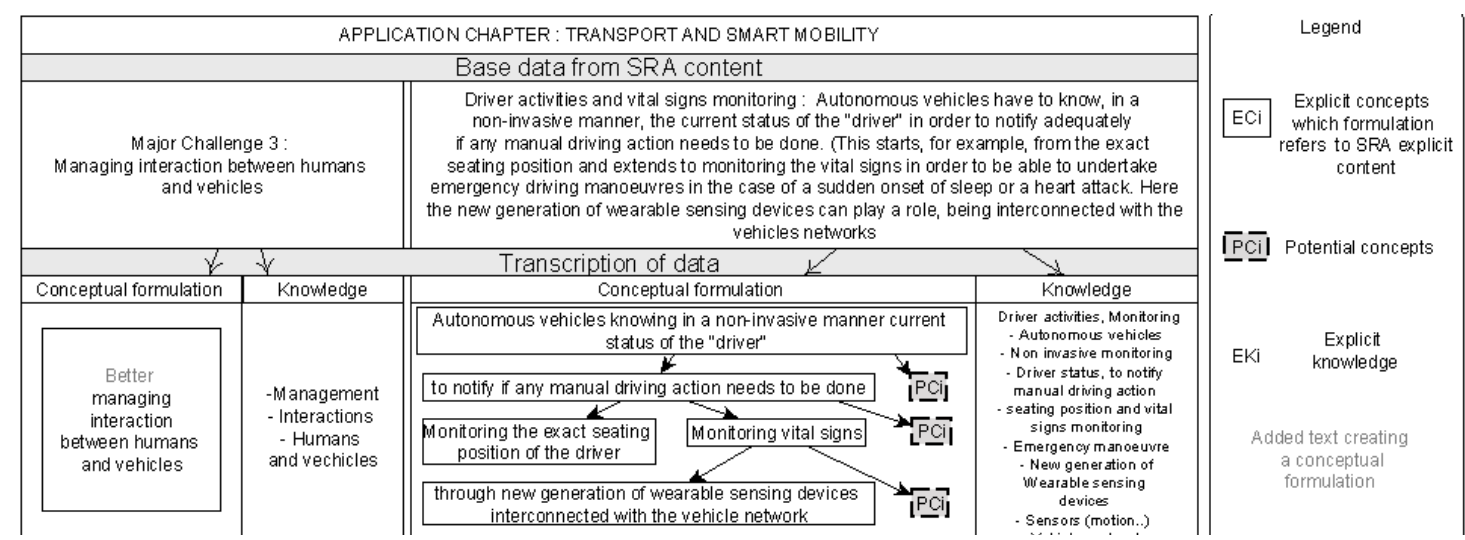

Figure 2. Example of ECS SRA transcription into concepts and knowledge at the sentence or paragraph level, revealing uncovered potential conceptual paths (PCi) in paragraphs

"better" to their initial formulation. Detailed paragraphs are transcribed into local trees of several ECi. It appears as first result that some uncovered potential paths (PCi) exist at the paragraph level since 
partitions are not complete with ECi according to $\mathrm{C}-\mathrm{K}$ theory. Indeed, the first partition level deals with the transition between autonomous driving and manual driving. "To notify if any manual driving action needs to be done" is the only path stated in the paragraph. C-K theory helps us to identify that designing different desirable paths is possible here. Thinking of potential examples reveals other solutions are possible as autonomous driving is able to take the lead and substitute itself for manual driving. That is why the partition level is not complete and thus we position a $\mathrm{PCi}$ on the diagram to notify it. The second partition level is also restrictive although two different paths are proposed: "Monitoring the exact seating position of the driver" and "monitoring vital signs". Nevertheless, other types of information could be of interest as the driver's vigilance level. In some cases, the ECS SRA itself clearly mentions the existence of those potential concepts at a partition level, when detailing on one hand an example and stating on the other hand that some other paths could be explored without detailing much further.

Once we have translated all the Major Challenge content, we have to link the different resulting concepts. Indeed, Figure 2 does not show the link between "Managing interaction between humans and vehicles" and "Autonomous vehicles knowing in a non-invasive manner the current status of the driver" and the other transcribed concepts that are presented in Figure 3 as ECi. As it can be seen, we are able to organise the knowledge basis according to all the EKi fragments, but the links between different ECi are not described in the Major Challenge. That is why we need to resort to ICi to organise them as Figure 3 shows it. In Figure 3, the links are created at the level of the Major Challenge, but they can also be created at the level of the chapter as in Figure 4. An example of ICi from Major Challenge 3 is "through a better direct interaction with vehicles" which is a mother concept shared by all the Major Challenge ECi and can be seen at the top of Figure 3. As it is not the only path that we could think of, we position PCi to indicate potential additional partitions could be found at a later stage. ICi could be added at any partition levels and they shed light on paths that were potentially unconsciously chosen by roadmap contributors.

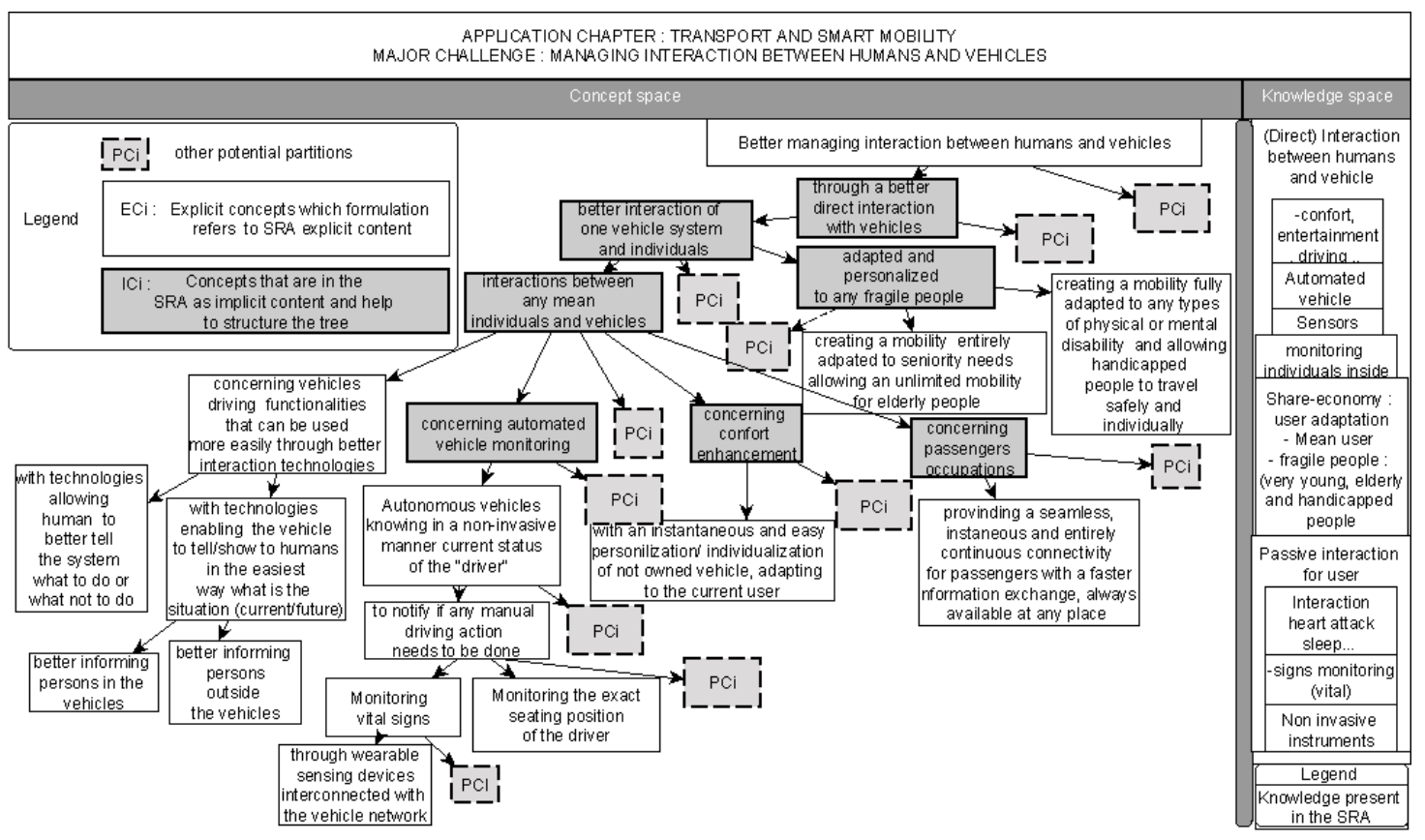

Figure 3. Creating a general concept-tree structure using ECS SRA ICi, revealing additional PCi not covered by the ECS SRA at the Major Challenge level

Regarding the ECS SRA chapter about ECS Process Technology, Equipment, Materials and Manufacturing, identification of innovation fields that are not present in the ECS SRA, was harder than for the application chapter about Transport and Smart Mobility. The text displays a structure which more closely reflects $\mathrm{C}-\mathrm{K}$ like partitions, and the tree architecture is quite entirely described in the document. As illustrated in Figure 4 which compares the two studied chapter structures, the ECS SRA presents a disparity among its chapters, according to C-K theory. 
Finally, to answer our first research question, this methodology allows us to pinpoint clearly where covered research areas are, and where some uncovered paths (PCi) are, in order to diagnose through discussion with ECS SRA contributors whether some innovative paths are not covered unintentionally. It appears the ECS SRA displays uncovered paths at the paragraph and major challenges level but also at the chapter level. Furthermore, the studied chapters present a disparity of transcription into C-K diagram.

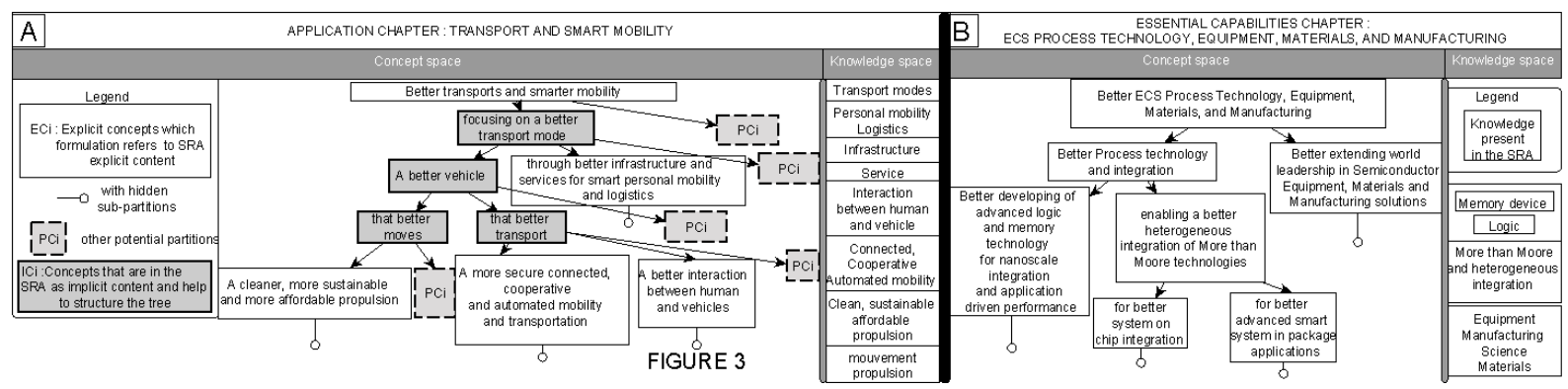

Figure 4. Heterogeneity of ECS SRA chapters: Each diagram represents an entire chapter where Major Challenges are transcribed into ECi. (A) The concept-tree structure elaboration of "Transport and Smart Mobility" chapter requires the use of ICi. (B) In contrast, the concept-tree structure elaboration of "ECS Process Technology, Equipment, Materials and Manufacturing" does not need to introduce implicit concepts as the explicit content of this chapter almost already constitutes a partition.

This study allows us to propose some guidance to the writing process of a Strategic Research Agenda. When first analysing the ECS SRA structure, we understood that the bullet points structure of the ECS SRA answered well all the diverse constraints of its elaboration. However, this highly condensed content, constituting a major part of the ECS SRA is not formulated with concepts. Instead, concepts which are present in the ECS SRA without needing any reformulation, are situated at a very detailed level, most of the time in paragraphs. Listed research questions do not bring to light what the current factors hampering innovation are, which would transform a topic into a concept, according to $\mathrm{C}-\mathrm{K}$ theory. That is related to the fact that very little knowledge is explicitly mentioned in the document, preventing a layman to identify clearly what is the implicit concept under a classification title such as "managing interaction between humans and vehicles". However, a scientific expert from this field can identify what is the related implicit knowledge of a topic and can transform it into a concept. Making explicit conceptual models used by experts while writing the document, would enable the reader to link the various listed elements. It would allow the possibility of better identifying consciously all the partition levels and concepts between sentence and paragraphs, in order to choose deliberately the most relevant coverage. Secondly, formulating the ECS SRA with more problem-oriented sentences and conceptual formulation rather than stating research questions would enable to explicit precisely current issues and address them through a wider scope of solutions. The goal is to prevent the ECS SRA focusing either on a hype subject or a dominant design, but rather systematically generating different approaches. Moreover, it was noted earlier that the ECS SRA sometimes used examples to explicit concepts. Using expansive examples (Agogué and al, 2014), modifying the object identity by adding unexpected attributes, help to generate non-intuitive pathways. Recent experiments based on a cognitive perspective showed these kind of examples significantly increase the resistance to fixation (Agogué and Cassotti, 2012) and must open more easily the pathway to potential additional concepts. This suggests that using expansive examples in an SRA elaboration could be an effective way to avoid fixation effects and increase the overall coverage.

\subsection{Adding knowledge to build more robust trees and enrich the ECS SRA scope}

To build a more robust tree, we include additional knowledge to our SRA raw transcription. Through this approach, we investigate, with different methodologies, how to enrich the ECS SRA scope.

\subsubsection{Investigating potential concepts (PCi) through logical rules}

First, we consider areas previously identified as potential concepts (PCi). New concepts are created through logical rules applied on surrounding explicit or implicit concepts sharing the same partition level. As an example, the new concept "better indirect interaction with vehicles" completes the 
partition level of the ICi: "better direct interaction with vehicles" using a complementary concept as presented in Figure 5. Such logical operations help new concepts to differentiate from the dominant design and potential new knowledge from external community may be needed to understand their implications. Thanks to this methodology, several different types of concepts are discovered. In the first place, new concepts (A) that are not present at all in the ECS SRA are created, as it is the case of our previous example. Some other concepts created through that approach are present in other ECS SRA chapters (B), which proves their relevance. Lastly, some concepts appear to be indirectly present in the ECS SRA (C). Indeed, at least one of their mother concepts, as well as some concepts from the same partition level, are ECi, but they are not themselves explicitly formulated in the document. Since the ECS SRA is intended as a guidance for project proposals, a way to control the relevance of the addition of such concepts, apart from their alignment with other ECS SRA chapters content, is to map project proposals on the concept-tree.

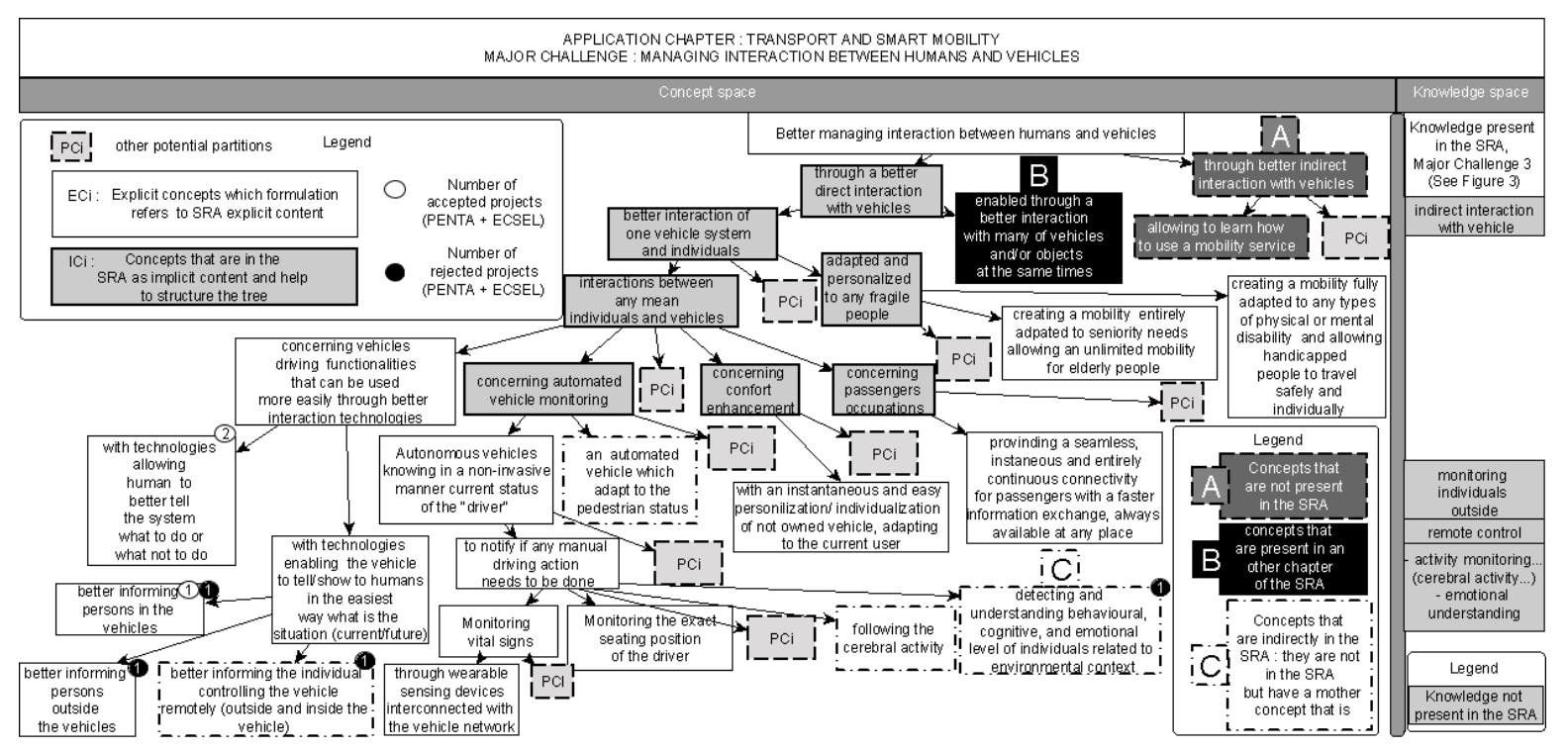

Figure 5. New concepts identification through PCi, using logical rules on ECi or ICi:

(A) Identification of new concepts, not present in the ECS SRA (B) Identification of concepts present in other ECS SRA chapters (C) Identification of concepts indirectly present

in the ECS SRA as they are not mentioned explicitly but at least one of their mother concepts is cited in the document as well as some concepts from the same partition level.

To test our diagrams, we map, onto the concepts-tree, projects proposals addressing the common subject-matter. Indeed, as was previously said, the ECS SRA is a cornerstone for the calls for projects of different programmes. Project proposals submitted to those programmes are considered through their publishable summary or project outline where their major concept often emerges. Then, projects are positioned on the concept-tree according to their central concept, as presented in Figure 5, where we make the additional distinction between funded and non-funded project proposals. For instance, we reviewed 92 projects proposals from ECSEL and PENTA programmes dealing with ECS Process Technology, Equipment, Materials and Manufacturing and 54 projects proposals dealing with Transport and Smart Mobility. Our interactions with members from ECSEL and PENTA allowed us to position correctly the different project proposals. Those projects are well scattered on the conceptual map in Figure 5. As a matter of fact, some of those projects are addressing our added concepts, which proves those concepts are relevant to the ECS SRA scope.

\subsubsection{Adding knowledge coming from various communities to Explicit Concepts (ECi)}

Another methodology consists in adding knowledge to an explicit concept. For instance, we look for additional knowledge on mobility coming from external communities, to tackle the chapter concept "ECS allowing better Transport and Smarter Mobility". The work of Amar (2018) is taken as a knowledge and conceptual resource for this chapter as it is a prospective view of mobility. Other experts are also consulted, and they reveal the importance of the multimodal aspect of mobility which is explored as a new concept. Indeed, they propose some paths to investigate it in Figure 6, which are compared later to the ECS SRA content. It appears that the ECS SRA mainly focuses on objects, and 
specifically on vehicle automation enabling future multimodality. For instance, mutualisation and synergy between objects are not addressed in the ECS SRA document. Nevertheless, some actors proposed project proposals addressing those concepts which proves the relevance of such investigations and may help to better structure diagrams following ECS SRA structure. Regarding the technical chapter about ECS Process Technology, Equipment, Materials and Manufacturing, we benefited from organized knowledge from different sources like ITRS and IRDS. In fact, those sources did not help to find disruptive branches while ITRS was proven to be of good coverage (Cogez and al, 2011, 2013).

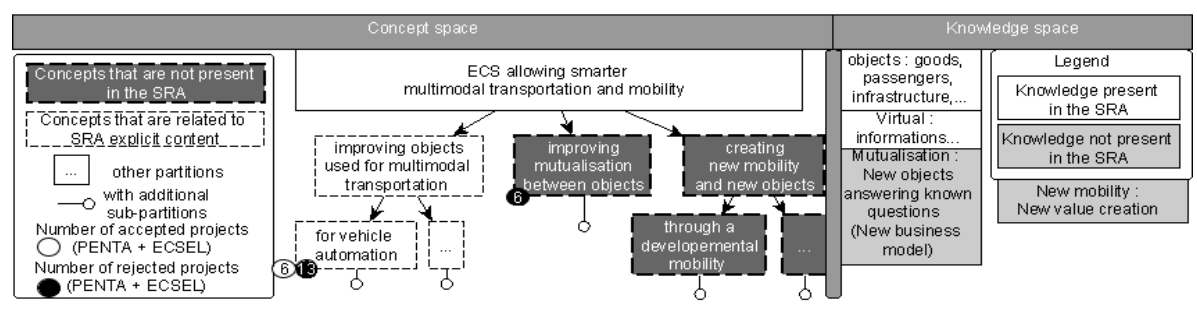

Figure 6. Exploration of the concept" ECS allowing smarter multimodal transportation and mobility". This concept that is not present in the Transport and Smart Mobility chapter was determined through added knowledge coming from external communities

This confirms that the ECS SRA technical chapter displays a large coverage which overlaps ITRS and IRDS content. However, this knowledge helps us to create a more structured knowledge basis allowing us to reorganize the global tree at the chapter level with partitions following $\mathrm{C}-\mathrm{K}$ theory criteria, as shown in Figure 7. Thus, this added knowledge allows us to better structure in terms of diagram the chapter content, enabling a wider vision of the chapter landscape and the projects distribution, while highlighting the ECS SRA uncovered areas that would be relevant to investigate further.

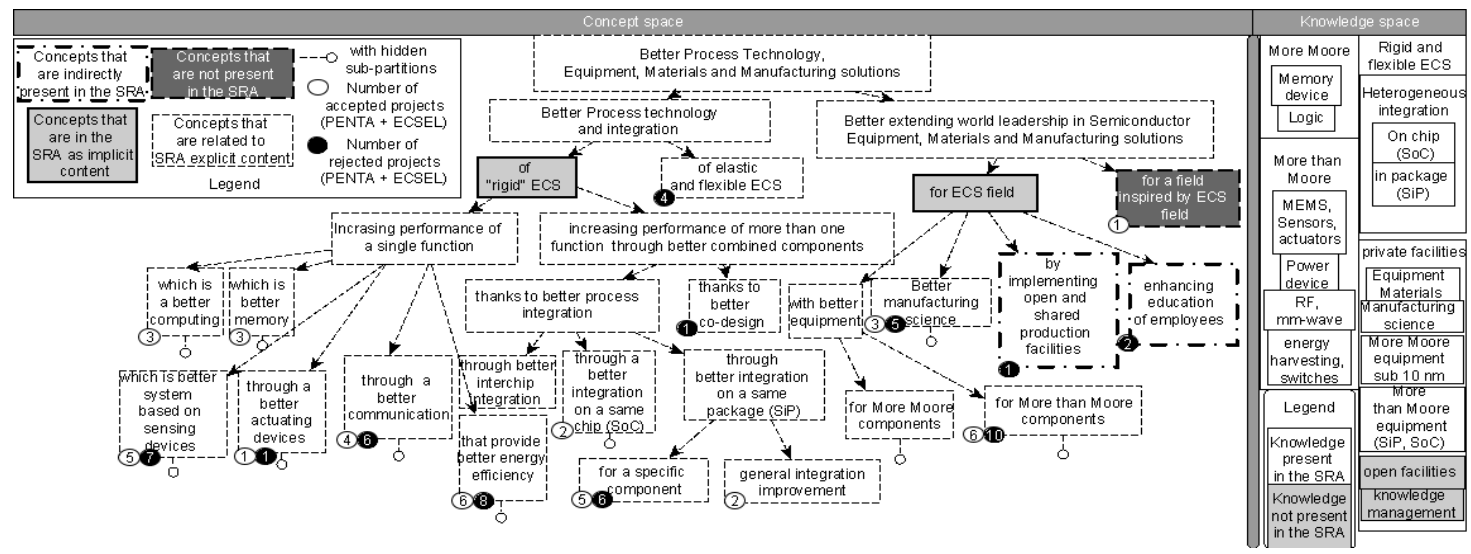

Figure 7. Excerpt from the ECS SRA referential diagram of the technical chapter including identification of uncovered areas and project proposals distribution.

\subsubsection{Generating new concepts to complete the ECS SRA scope}

The last approach consists in using knowledge of the ECS SRA to generate new concepts. So far, we considered actors as neutral in our study. Nevertheless, the fact that the ECS SRA is the basis for funding decisions of European collaborative research projects plays a great role in its content. But this aspect does not necessarily contribute to generate new desirable concepts. Thus, we proposed to use this strategic knowledge to force the generation of such concepts like for example "Memory devices suited for Artificial Intelligence needs and for which manufacturing can be brought back to Europe". The European dimension is here used to generate new concepts aligned with the ECS SRA goal, which could then be explored through C-K theory. Thus, this method help us to explore concepts out of the dominant design. The exploration of such a concept through C-K theory in Figure 8, highlighted the need to merge knowledge from different ECS SRA chapters which may explain they are not present in the current document.

To conclude, we investigated three different methods to identify and explore uncovered innovation paths that are not in the dominant design of the field. For that we use additional knowledge coming from internal or external experts. Those concepts can eventually be themselves explored through $\mathrm{C}-\mathrm{K}$ theory, potentially involving experts from various communities. The relevance of such concepts was then verified through the 
mapping of projects proposals proving some actors consider those paths to be of interest. Besides, this mapping could prove useful to manage project portfolios of collaborative projects as Figure 7 shows.

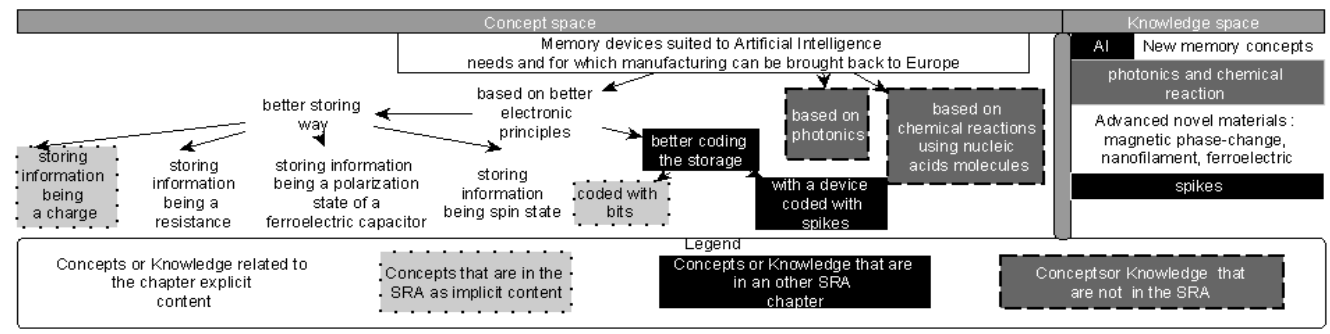

Figure 8. Exploration of a new concept merging knowledge from several ECS SRA chapters

\section{CONTRIBUTION AND DISCUSSION}

Our methodology, based on a C-K framework, provided us with the ability to diagnose whether there are some unintentionally uncovered areas in SRA. Discussion with experts allowed us to prove the relevance of our methodology, and then to identify new value-added innovation areas, that were not mentioned in the document, in order to enlarge its scope. Project proposal mapping confirmed the adequacy of newly identified innovation paths. Thus, this work provides support to make more explicit strategic decisions made on SRA selected coverage.

However, despite the sample variety and quality of our material, further researches, including a design framework, are needed on distinctive SRA. Indeed, it is the technology forecasting literature which is mostly interested in the elaboration process of agendas. On the contrary, instead of being used to create SRA guidelines, engineering design usually deals with SRA topics once they have been defined. Nevertheless, the establishment of such strategies requires a design effort in order to formulate correctly the technology directions. Today, we believe engineering design can tackle those questions related to SRA elaboration and scope. For instance, it could contribute to increase the relevant coverage of an SRA, revealing a tool that manages the risks linked to unidentified innovation paths and supports collective action aiming exploration of promising innovations. Few studies (Cogez and al, 2013) have implemented design approaches on roadmaps assessment, and we first pave the way to SRA analysis. But our approach also echoes more general aspects of research and the way design theory could help to consider formulation of calls for projects in order to spur more innovative solutions as well as the way to manage scientific portfolio through conceptual maps, for instance.

This work also reveals its usefulness for various kind of actors which raises the question of its implementation as a service in different contexts. Table 1 presents a variety of services using this methodology which could be offered to different types of clients.

Table 1. Services that could be provided based on the methodologies of this study

\begin{tabular}{|c|c|c|c|c|c|c|}
\hline CLIENTS & $\begin{array}{c}\text { SRA } \\
\text { contributors }\end{array}$ & $\begin{array}{c}\text { Project } \\
\text { evaluators }\end{array}$ & $\begin{array}{c}\text { Potential } \\
\text { consortiums }\end{array}$ & $\begin{array}{c}\text { Existing } \\
\text { consortiums }\end{array}$ & \begin{tabular}{|c|} 
Funding \\
programmes
\end{tabular} & $\begin{array}{c}\text { Public } \\
\text { Authorities }\end{array}$ \\
\hline OUTPUTS & $\begin{array}{l}\text { - Proposition } \\
\text { of new } \\
\text { methodologies } \\
\text { for chapter } \\
\text { elaboration } \\
\text { - Explicit the } \\
\text { selected SRA } \\
\text { coverage }\end{array}$ & $\begin{array}{c}\text { - Suggesting } \\
\text { improvements } \\
\text { in proposal } \\
\text { scope and/or } \\
\text { constitution } \\
\text { membership }\end{array}$ & $\begin{array}{l}\text { - Exploration to } \\
\text { extend the use } \\
\text { cases and } \\
\text { application } \\
\text { domains of a } \\
\text { proposed concept } \\
\text { - Identification of } \\
\text { potential partners }\end{array}$ & $\begin{array}{l}\text { - Promoting } \\
\text { further the } \\
\text { project after } \\
\text { its end } \\
\text { through } \\
\text { diverse } \\
\text { explorations }\end{array}$ & $\begin{array}{l}\text { - SRA } \\
\text { referential } \\
\text { map with } \\
\text { projects } \\
\text { distribution }\end{array}$ & $\begin{array}{l}\text { - SRA } \\
\text { referential } \\
\text { map with } \\
\text { projects } \\
\text { distribution } \\
\text { of the } \\
\text { considered } \\
\text { country/ } \\
\text { Europe }\end{array}$ \\
\hline
\end{tabular}

\section{REFERENCES}

Agogué, M., and Cassotti, M. (2012), "Theory-driven experiments: Modeling and testing fixation and stimulation effects on creativity", 5th Paris Workshop of the Design Theory SIG, Paris, France, January 30, 2012

Agogué, M., Kazakçi, A., Hatchuel, A., Le Masson, P., Weil, B., Poirel, N., and Cassotti, M. (2014), "The Impact of Type of Examples on Originality: Explaining Fixation and Stimulation Effects", The Journal of Creative Behavior, Vol. 48 No. 1, pp. 1-12. http://doi.org/10.1002/jocb.37 
Agogué, M., Le Masson, P., and Robinson, D.K.R. (2012), “Orphan innovation, or when path-creation goes stale: a design framework to characterise path-dependence in real time”, Technology Analysis \& Strategic Management, Vol. 24 No. 6, pp. 603-616. http://doi.org/10.1080/09537325.2012.693672

Amar, G. (2018), "Dealing with the Future: General Considerations and the Case of 'Mobility", In: Subrahmanian E. Odumosu T., Tsao J. (eds), Engineering a Better Future, Springer, Cham, pp.197-200, https://doi.org/10.1007/978-3-319-91134-2_16

Barker, D., and Smith, D. (1995), “Technology foresight using roadmaps”, Long Range Planning, Vol. 28 No. 2 , pp. 21-28. http://doi.org/10.1016/0024-6301(95)98586-H

Chen, M., Aknin, P., Lagadec, L.-R., Laousse, D., Le Masson, P., and Weil, B. (2017), "Designing the missing link between science and industry: Organizing partnership based on dual generativity", International Conference on Engineering Design, Vol. 4, pp. 307-316

Cogez and al. (2019), Electronic Components \& Systems Strategic Research Agenda 2019, available at https://aeneasoffice.org/strategy/documents/

Cogez, P., Felk,Y., Le Masson, P., and Weil, B. (2011), “Absorptive capacity for radical innovation: a case study in the semiconductor industry”, IEEE International Technology Management Conference, San Jose, California.

Cogez, P., Kokshagina, O., Le Masson, P., and Weil, B. (2013), "Industry-Wide Technology Roadmapping in Double Unknown - The Case of the Semiconductor Industry", 2013 IEEE International Technology Management Conference \& 19th ICE Conference (ITMC), The Hague, 6/2013, IEEE, pp. 1-13. http://doi.org/10.1109/ITMC.2013.7352611

David, P.A. (1985), "Clio and the economics of QWERTY”, American Economic Review, Vol. 75 No. 2, pp. 332 337.

Galvin, R. (1998), “Science Roadmaps”, Science, Vol. 280 No. 5365, pp. 803-803. https://doi.org/10.1126/science.280.5365.803a

Garud, R., and Karnoe, P. (2001), Path Dependence and Creation, Series in Organization and Management, Taylor \& Francis

Hatchuel, A., and Weil, B. (2009), "C-K design theory: An advanced formulation”, Research in Engineering Design, Vol. 19 No. 4, pp. 181-192. http://doi.org/10.1007/s00163-008-0043-4

IRDS (2017), IRDS International Roadmap for Device and Systems, available at: (https://irds.ieee.org/roadmap-2017) $(14 / 11 / 2018)$

ITRS (2001-2015), ITRS International Technology Roadmap for Semiconductors, available at: (http://www.itrs2.net/itrs-reports.html) (14/11/2018)

Kostoff, R. N., and Schaller, R. R. (2001), "Science and technology roadmaps", IEEE Transactions on Engineering Management, Vol. 48 No. 2, pp. 132-143. https://doi.org/ 10.1109/17.922473

Lee, S., and Park, Y. (2005), "Customization of technology roadmaps according to roadmapping purposes: Overall process and detailed modules", Technological Forecasting and Social Change, Vol. 72 No. 5, pp. 567-583. https://doi.org/10.1016/j.techfore.2004.11.006

Le Masson, P., Weil, B., and Hatchuel, A. (2017), Design Theory, Springer International Publishing, Cham. https://doi.org/10.1007/978-3-319-50277-9_2

Le Masson, P., Weil, B., Hatchuel, A., and Cogez, P. (2012), "Why aren’t they locked in waiting games? Unlocking rules and the ecology of concepts in the semiconductor industry", Technology Analysis \& Strategic Management, Vol. 24 No. 6, pp. 617-630.

Phaal, R., Farrukh, C.J.P., and Probert, D.R. (2004), “Technology roadmapping--A planning framework for evolution and revolution", Technological Forecasting and Social Change, Vol. 71 No. 1, pp. 5-26. https://doi.org/10.1016/S0040-1625(03)00072-6.

Robinson, D.K.R., and Propp, T. (2008), "Multi-path mapping for alignment strategies in emerging science and technologies", Technological Forecasting and Social Change, Vol. 75 No. 4, pp. 517-538. https://doi.org/10.1016/j.techfore.2008.02.002

Rosenberg, N. (1976), "The Direction of Technological Change: Inducement Mechanisms and Focusing Devices", Economic Development and Cultural Change, Vol.18 No.1, pp.1-24. https://doi.org/10.1086/450399

Vourc'h, G., Brun, J., Ducrot, C., Cosson, J.-F., Le Masson, P., and Weil, B. (2018), 'Using design theory to foster innovative cross-disciplinary research: Lessons learned from a research network focused on antimicrobial use and animal microbes' resistance to antimicrobials", Veterinary and Animal Science, Vol. 6, pp. 12-20. http://doi.org/10.1016/j.vas.2018.04.001

Willyard, C.H., and McClees, C. (1987), "Motorola's technology roadmap process", Research Management, Vol. 30 No. 5, pp.13-19. https://doi.org/10.1080/00345334.1987.11757057

\section{ACKNOWLEDGMENTS}

We thank all the experts involved in our study for their guidance and their contribution. 